\title{
Prognostic significance of L-type amino-acid transporter I expression in surgically resected pancreatic cancer
}

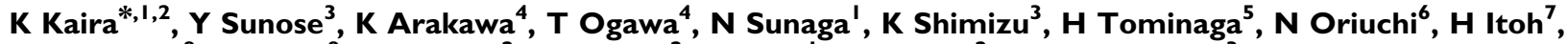 S Nagamori ${ }^{8}$, Y Kanai ${ }^{8}$, A Segawa ${ }^{2}$, M Furuya ${ }^{2}$, M Mori', T Oyama ${ }^{2}$ and I Takeyoshi ${ }^{3}$}

'Department of Medicine and Molecular Science, Gunma University Graduate School of Medicine, Showa-machi, Maebashi, Gunma 37 I-85 I I, Japan; ${ }^{2}$ Department of Diagnostic Pathology, Gunma University Graduate School of Medicine, Showa-machi, Maebashi, Gunma 37I-85 I I, Japan; ${ }^{3}$ Department of Thoracic and Visceral Organ Surgery, Gunma University Graduate School of Medicine, Showa-machi, Maebashi, Gunma 37I-85I I, Japan; ${ }^{4}$ Department of Surgery, Maebashi Red Cross Hospital, Asahi-cho, Maebashi, Gunma, Japan; ${ }^{5}$ Department of Molecular Imaging, Gunma University Graduate School of Medicine, Showa-machi, Maebashi, Gunma 37 I-85 I I, Japan; 'epartment of Diagnostic Radiology and Nuclear Medicine, Gunma University Graduate School of Medicine, Showa-machi, Maebashi, Gunma 37 I-85II, Japan; ${ }^{7}$ Department of Pathology, Maebashi Red Cross Hospital, Asahi-cho, Maebashi, Gunma, Japan; ${ }^{8}$ Division of Bio-system Pharmacology, Graduate School of Medicine, Osaka University, Osaka, Japan

BACKGROUND: The expression of L-type amino-acid transporter I (LATI) is tumour-specific and has been shown to have essential roles in cell growth and survival. However, little is known regarding the clinical significance of LATI expression in pancreatic cancer. This study was conducted to determine the prognostic significance of LATI expression.

METHODS: A total of 97 consecutive patients with surgically resected pathological stage I-IV pancreatic ductal adenocarcinoma were retrospectively reviewed. Tumour sections were stained by immunohistochemistry for LATI, CD98, Ki-67 and vascular endothelial growth factor (VEGF), and microvessel density was determined by CD34 and p53.

RESULTS: L-type amino-acid transporter I and CD98 were highly expressed in 52.6\% (5I/97) and 56.7\% (55/97) of cases, respectively $(P=0.568)$. The expression of LATI within pancreatic cancer cells was significantly associated with disease stage, tumour size, Ki-67, VEGF, CD34, p53 and CD98. L-type amino-acid transporter I expression was confirmed to be a significant prognostic factor for predicting poor outcome by multivariate analysis.

CONCLUSION: L-type amino-acid transporter I expression is a promising pathological marker for the prediction of outcome in patients with pancreatic cancer.

British Journal of Cancer (2012) 107, 632-638. doi:10.1038/bjc.2012.310 www.bjcancer.com

Published online 17 July 2012

(c) 2012 Cancer Research UK

Keywords: LATI; CD98; pancreatic cancer; amino-acid transporter; prognostic factor; Ki-67

Pancreatic cancer has a poor prognosis and is the leading cause of cancer death, although the disease represents only $3 \%$ of all tumours (Jemal et al, 2008). Surgical resection remains the only potentially curative therapeutic option. At the time of initial diagnosis, only a minority of patients with pancreatic cancer are at a disease stage that can still potentially be cured by resection (Geer and Brennan, 1993). Even if a potentially curative resection can be performed, the 5-year overall survival (OS) is low at 10-25\% (Geer and Brennan, 1993; Cameron et al, 2006). To improve patient outcome, clinical markers that may predict the prognosis and response to the specific therapy should be established. Current prognostic biomarkers for curatively resected pancreatic cancer are thought to be lymph node status, tumour type, histological grade, tumour staging and performance status (Geer and Brennan, 1993; Delcore et al, 1996; Cameron et al, 2006; Hellan et al, 2008). However, no established clinical markers have correlated with outcome and therapeutic response in patients with pancreatic cancer.

Amino-acid transporters are essential for the growth and proliferation of normal cells and transformed cells

*Correspondence: Dr K Kaira; E-mail: kkaira1970@yahoo.co.jp

Received 23 April 2012; revised 14 May 2012; accepted I8 June 2012; published online 17 July 2012
(Christensen, 1990; McGivan and Pastor-Anglada, 1994). L-type amino-acid transporter 1 (LAT1) is an L-type amino-acid transporter that transports large neutral amino acids, such as leucine, isoleucine, valine, phenylalanine, tyrosine, tryptophan, methionine and histidine (Kanai, et al, 1998; Yanagida et al, 2001). L-type amino-acid transporter 1 requires covalent association with the heavy chain of 4F2 cell surface antigen (CD98) for its functional expression in the plasma membrane (Kanai et al, 1998). L-type amino-acid transporter 1 has been closely associated with cancerous or proliferative cells, and previous studies have shown that LAT1 is highly expressed in proliferating tissues, many tumour cell lines (T24 bladder carcinoma cells, RERF-LC-MA lung small-cell carcinoma cells and HeLa uterine cervical carcinoma cells) and primary human tumours (Yanagida et al, 2001; Kobayashi et al, 2005; Nawashiro et al, 2006; Nakanishi et al, 2007; Kaira et al, 2008; Sakata et al, 2009; Ichinoe et al, 2011). Recent studies have demonstrated that the level of LAT1 expression is a significant factor that indicates poor prognosis in various human cancers, including lung cancer (Kaira et al, 2008), urologic cancer (Nakanishi et al, 2007), brain tumours (Nawashiro et al, 2006), prostatic cancer (Sakata et al, 2009) and gastric cancer (Ichinoe et al, 2011). L-type amino-acid transporter 1 provides cancer cells with the amino acids that are essential not 
only for protein synthesis but also for the stimulation of cancer cell growth via mammalian targeting of rapamycin (Fuchs and Bode, 2006).

Although LAT1 has been described to have a crucial role in the pathogenesis of human neoplasms, the clinical significance of LAT1 expression in pancreatic cancer remains unclear (Fuchs and Bode, 2006; Nawashiro et al, 2006; Nakanishi et al, 2007; Kaira et al, 2008; Sakata et al, 2009; Ichinoe et al, 2011). Previous studies have demonstrated that LAT1 expression is closely associated with CD98, cell proliferation (Ki-67-labelling index), the cell cycle regulator p53 and angiogenesis in patients with thoracic tumours (Kaira et al, 2011a, 2011b). Therefore, in this study, the expression level of LAT1 protein was assessed in resected tissue specimens and correlated with the outcome of patients with pancreatic cancer. In addition, the correlation between LAT1 expression and CD98, the Ki-67-labelling index, p53, and angiogenic markers, such as vascular endothelial growth factor (VEGF) expression and microvessel density (determined by $\mathrm{CD} 34)$, was examined.

\section{PATIENTS AND METHODS}

\section{Patients}

We analysed 113 consecutive patients with pancreatic ductal adenocarcinoma (AC) who underwent surgical resection at Gunma University Hospital and Maebashi Red Cross Hospital between July 1995 and March 2011. Nine patients who received induction chemotherapy or radiation therapy were excluded. The specimens from seven patients were not available. Thus, a total of 97 patients were analysed in the study. The study protocol was approved by the institutional review board.

The age of the patients ranged from 25 to 86 years, and the median age was 67 years. None of the patients had received neo-adjuvant chemotherapy. All surgical specimens were reviewed and classified according to the WHO classification by an experienced pathologist who was unaware of clinical or imaging findings. Pathological tumour-node-metastasis stages were established using the International System for Staging Pancreatic Cancer adopted by the American Joint Committee on Cancer and the Union Internationale Centre le Cancer (Sobin et al, 2009). In all cases, magnetic resonance cholangiopancreatography and endoscopic retrograde cholangiopancreatography were performed before surgical resection, and intrapancreatic common bile duct carcinoma (cholangiocarcinoma) and ampullary carcinoma were excluded from this study. Histologically, all patients were diagnosed with AC. Of the total patients, 18, 40, 32 and 7 had stage I, II, III and IV tumours, respectively. Postoperative adjuvant chemotherapy with gemcitabine, S-1 (Taiho Pharmaceutical Co., Ltd, Tokyo, Japan) and oral administration of tegafur (a fluorouracil derivative drug) were administered to 31,15 and 1 patients, respectively. Intraoperative therapy was not performed on any patient. The day of surgery was considered the starting day for measuring postoperative survival. The followup duration ranged from 6 to 164 months (median, 14 months).

A control group of patients with benign pancreatic lesions was also analysed. Immunohistochemical staining of samples from 18 patients with surgically resected benign pancreatic diseases was performed and compared with that of pancreatic cancer. The histology of resected benign pancreatic lesions was as follows: four patients with intraductal papillary-mucinous adenoma, two patients with solid-pseudopapillary tumour, two patients with serous cystadenoma, three patients with pseudocyst, two patients with lymphoplasmacytic sclerosing pancreatitis, two patients with chronic sclerosing pancreatitis, one patient with inflammatory pseudotumour, one patient with pancreatic endocrine cell tumour (insulinoma) and one patient with abscess of pancreas.

\section{Immunohistochemical staining}

L-type amino-acid transporter 1 expression was determined by immunohistochemical staining with a LAT1 antibody $\left(2 \mathrm{mg} \mathrm{ml}^{-1}\right.$, anti-human monoclonal mouse antibody, 4A2, provided by $\mathrm{Dr} \mathrm{H}$ Endou (J-Pharma, Tokyo, Japan), dilution; 1:3200). The production and characterisation of the LAT1 antibody has previously been described (Sakata et al, 2009). The CD98 antibody is an affinity-purified rabbit polyclonal antibody (Santa Cruz Biotechnology Inc., Santa Cruz, CA, USA, 1:100 dilution) raised against a peptide mapping to the carboxy terminus of CD98 of human origin. The detailed protocol for immunostaining has been published elsewhere (Kaira et al, 2008). L-type amino-acid transporter 1 and CD98 expression was considered positive only if distinct membrane staining was present. The LAT1 and CD98 expression scores were assessed by the extent of staining as follows: $1, \leqslant 10 \%$ of tumour area stained; 2, 11-25\% stained; 3, 26-50\% stained; and $4, \geqslant 51 \%$ stained. The staining intensity was not considered when staining was assessed. The tumours in which stained tumour cells were scored as 3 or 4 were defined as high expression.

For VEGF, CD34, Ki-67 and p53, immunohistochemical staining was performed according to the procedures described in previous reports (Kaira et al, 2009, 2010a). The following antibodies were used: a monoclonal antibody against VEGF (Immuno-Biological Laboratories Co. Ltd, Fujioka, Japan, 1:100 dilution); mouse monoclonal antibodies against CD34 (Nichirei, Tokyo, Japan, 1:800 dilution), Ki-67 (Dako, Glostrup, Denmark, 1:40 dilution) and p53 (D07; Dako, 1:50 dilution). The expression of VEGF was quantitatively assessed according to the percentage of immunoreactive cells among a total of 1000 neoplastic cells. The number of CD34-positive vessels was counted in four selected hot spots in a $\times 400$ field $\left(0.26 \mathrm{~mm}^{2}\right.$ field area). Microvessel density was defined as the mean count of microvessels per $0.26 \mathrm{~mm}^{2}$ field area. The median rate of VEGF positivity and the median number of CD34positive vessels were evaluated, and the tumours in which stained tumour cells represented more than each median value were defined as high expression. For p53, microscopic examination for the nuclear reaction product was performed and scored. Based on a previous report (Kaira et al, 2010a), p53 expression in greater than $10 \%$ of the tumour cells was defined as positive expression. For Ki-67, a highly cellular area of the immunostained sections was evaluated. All epithelial cells with nuclear staining of any intensity were defined as high expression. Approximately 1000 nuclei were counted on each slide. Proliferative activity was assessed as the percentage of Ki-67-stained nuclei (Ki-67-labelling index) in the sample. The median value for the Ki-67-labelling index was evaluated, and the tumour cells with greater than the median value were defined as high expression. The sections were assessed using a light microscope in a blinded fashion by at least two of the authors.

\section{Statistical analysis}

Probability values of $<0.05$ indicated a statistically significant difference. Fisher's exact test was used to examine the association between two categorical variables. The correlation between different variables was analysed using the nonparametric Spearman's rank test. Follow-up for these 97 patients was conducted using the patient medical records. The Kaplan-Meier method was used to estimate survival as a function of time, and survival differences were analysed by the log-rank test. Overall survival was determined as the time from tumour resection to death from any cause. Progression-free survival (PFS) was defined as the time between tumour resection and the first disease progression or death. Multivariate analyses were performed using a stepwise Cox proportional hazards model to identify independent prognostic factors. Statistical analyses were performed using JMP 8 (SAS Institute Inc., Cary, NC, USA) for Windows. 
634

\section{RESULTS}

\section{Immunohistochemical analysis}

The immunohistochemical analysis of the biomarkers was performed on the 97 primary lesions with pancreatic cancer and 18 resected lesions with benign pancreatic diseases. Figure 1 represents the immunohistochemical staining of LAT1 and CD98 expression in patients with pancreatic cancer. L-type amino-acid transporter 1 immunostaining was detected in carcinoma cells in the tumour tissues and was localised predominantly on the plasma membrane. All positive cells revealed strong membranous LAT1 immunostaining. Cytoplasmic staining was rarely observed. In the 97 patients with pancreatic cancer, high LAT1 and CD98 expression was recognised in 52.6\% (51/97) and 56.7\% (55/97) of cases, respectively $(P=0.568)$. Among the 18 patients with benign pancreatic diseases, high LAT1 and CD98 expression was observed in $0 \%(0 / 18)$ and $44.4 \%(8 / 18)$ of cases, respectively $(P=0.003)$. The rate of high LAT1 and CD98 expression was significantly higher in pancreatic cancer than in benign pancreatic diseases (LAT1, $P<0.001$; CD98, $P=0.026$ ). The average LAT1 and CD98 expression scores in pancreatic cancer were $2.4 \pm 0.9$ and $2.6 \pm 0.8$ on a scale of $1-4$, respectively $(P=0.232)$; these scores in benign pancreatic diseases were $1.6 \pm 0.5$ and $2.4 \pm 1.0$, respectively $(P=0.002)$. The LAT1 expression scores were significantly higher in pancreatic cancer than in benign diseases $(P<0.001)$, but the CD98 expression scores were not significantly different between malignant and benign lesions $(P=0.528) \quad$ (Supplementary Figure A1, online only). Among the 51 patients with high LAT1 expression (score of 3 or 4), 39 (76.5\%) displayed high expression of CD98 (score of 3 or 4) and 12 displayed low expression (6 patients with a score of 2 and 6 patients with a score of 1 ) (Figure 1A). In these six patients with a score of 1 , no evidence of CD98 immunostaining was present.

The median rate of VEGF positivity was $40 \%$ (range, 5-80), and the value of $40 \%$ was chosen as a cutoff point. The median number of CD34 was 26 (range, 3-60), and the value of 26 was chosen as a cutoff point. The median value for the Ki-67-labelling index was $27 \%$ (range, 3-68), and the value of $27 \%$ was chosen as a cutoff point. Positive p53 expression was recognised in $40.2 \%$ (39/97) of cases. Table 1 shows the comparison of these biomarkers between pancreatic cancers and benign lesions. The rate of high expression or positivity of these biomarkers was significantly higher in pancreatic cancers than in benign pancreatic lesions.

\section{Demographics of patients according to LAT1 expression}

The demographic distribution of the other variables according to LAT1 expression is listed in Table 2. A statistically significant difference in disease stage, $\mathrm{T}$ factor, lymphatic permeation, vascular invasion and expression of CD98, Ki-67, VEGF, CD34 and 533 was observed between samples with high LAT1 and low LAT1 expression.
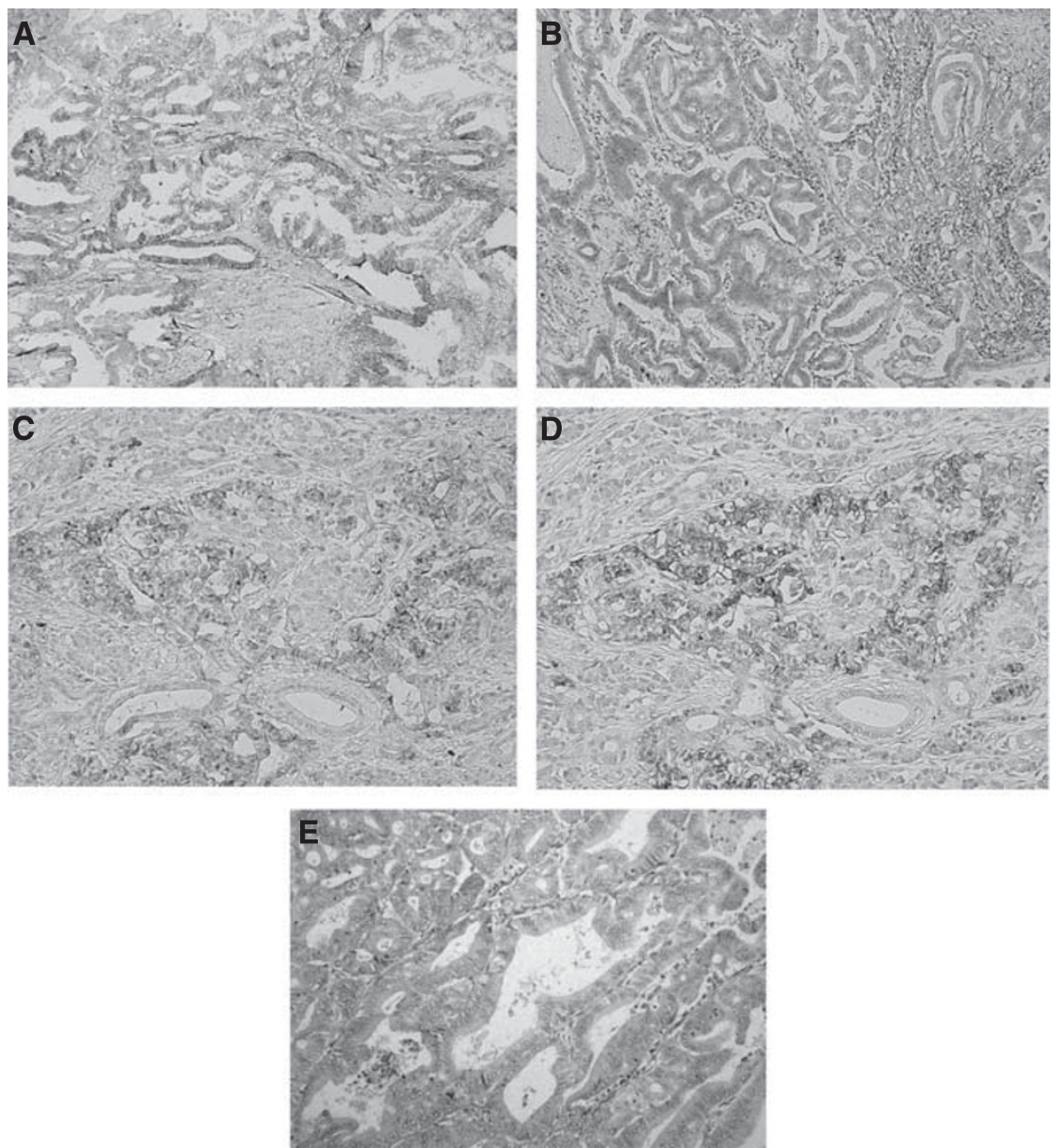

Figure I Immunohistochemical staining of tissue from a 60-year-old man with pancreatic ductal AC (p-stage IB). L-type amino-acid transporter I (A) immunostaining (score 4) displays a membranous immunostaining pattern but no evidence of CD98 (B) immunostaining (score I). Immunostaining of LAT I (C) and CD98 (D) in tissue from a 67-year-old man with pancreatic ductal AC (p-stage IIIB) showed a membranous immunostaining pattern in nearly the same area. The LATI and CD98 scores were 3 and 3, respectively. (E) shows negative staining for LATI in a patient with pancreatic ductal AC. 


\section{Correlation between LAT1 expression and different variables}

Using Spearman's rank correlation, a significant correlation was observed between LAT1 and CD98, Ki-67, VEGF, CD34 and tumour size (Table 3). Among the 18 patients with benign pancreatic diseases, the expression of LAT1 significantly correlated with CD98 expression $(\gamma=0.541, P=0.020)$ but not with VEGF $(\gamma=0.261, \quad P=0.296), \quad$ Ki-67 $\quad(\gamma=0.175, \quad P=0.488) \quad$ or $\quad \mathrm{CD} 34$ $(\gamma=0.293, P=0.238)$.

\section{Different variables and survival analysis}

The 5-year and 2-year survival rates and median survival time for all patients were $30.8 \%, 40.8 \%$ and 18.0 months (95\% confidence interval 12.9-24.6), respectively. Among all of the patients, 65 experienced postoperative recurrence, 26 received systemic chemotherapy with gemcitabine or S-1, and 53 died owing to disease progression. Tables 4 and 5 show the univariate and multivariate analysis of all patients $(n=97)$. In the univariate analysis, disease stage, lymphatic permeation, vascular invasion

Table I Comparison of different variables between pancreatic cancers and benign lesions

\begin{tabular}{|c|c|c|c|}
\hline \multirow[b]{2}{*}{ Variables } & \multicolumn{3}{|c|}{$\begin{array}{l}\text { Rate of high expression or } \\
\text { positivity (\%) in various biomarkers }\end{array}$} \\
\hline & $\begin{array}{l}\text { Pancreatic } \\
\text { cancers } \\
(n=97)\end{array}$ & $\begin{array}{c}\text { Pancreatic } \\
\text { benign } \\
\text { lesions }(n=18)\end{array}$ & $P$-value \\
\hline Age $(\leqslant 65 />65)$ & $(43 / 54)$ & $(10 / 8)$ & 0.445 \\
\hline Gender (male/female) & $(48 / 49)$ & $(7 / 11)$ & 0.451 \\
\hline LATI (high/low) & $52.6 \%(5 \mid / 97)$ & $0 \%(0 / 18)$ & $<0.001$ \\
\hline CD98 (high/low) & $56.7 \%(55 / 97)$ & $44.4 \%(8 / / 8)$ & 0.026 \\
\hline Ki-67 (high/low) & $49.5 \%(48 / 97)$ & $5.5 \%(1 / 18)$ & $<0.001$ \\
\hline VEGF (high/low) & $44.3 \%(43 / 97)$ & $16.7 \%(3 / 18)$ & 0.035 \\
\hline CD34 (high/low) & $49.5 \%$ (48/97) & $22.2 \%(4 / 18)$ & 0.040 \\
\hline p53 (positive/negative) & $40.2 \%(39 / 97)$ & $0 \%(0 / 18)$ & $<0.001$ \\
\hline
\end{tabular}

Abbreviations: LATI = L-type amino-acid transporter I; VEGF = vascular endothelial growth factor. Bold values indicate a statistically significant difference. and the expression of LAT1, Ki-67 and VEGF were significantly associated with poor PFS and OS. CD98 expression yielded a statistically significant difference in OS. Figure 2 shows the Kaplan-Meier survival curve in patients with high and low LAT1 and CD98 expression. Based on the results of the univariate log-rank test, we screened prognostic factors with a cutoff of $P<0.05$. Multivariate analysis confirmed that LAT1, Ki-67 and disease stage were independent prognostic factors for predicting poor OS. However, no significant independent prognostic factor was observed in the multivariate analysis of PFS.

Next, we performed survival analysis based on the LAT1 expression status. Multivariate analysis confirmed that disease stage and $\mathrm{Ki}-67$ were independent prognostic factors for predicting poor PFS and OS in 51 patients with high LAT1 expression (Supplementary Table A1, online only). However, in 46 patients with low LAT1 expression, Ki-67 was confirmed to be a significant prognostic factor for predicting poor PFS by multivariate analysis (Supplementary Table A2, online only).

\section{DISCUSSION}

This study is the first to evaluate the clinical significance of LAT1 expression in pancreatic cancer and clearly demonstrated that the expression of LAT1 was a significant independent factor for

Table 3 Correlation between LATI and other biomarkers in pancreatic cancer

\begin{tabular}{llc}
\hline Biomarkers & Spearman $\boldsymbol{\gamma}(\mathbf{9 5} \% \mathbf{C l )}$ & $\boldsymbol{P}$-value \\
\hline CD98 & $0.433(0.251-0.586)$ & $<\mathbf{0 . 0 0 1}$ \\
Ki-67 & $0.498(0.326-0.638)$ & $<\mathbf{0 . 0 0 ~ I}$ \\
VEGF & $0.538(0.374-0.669)$ & $<\mathbf{0 . 0 0 ~ I}$ \\
CD34 & $0.357(0.162-0.524)$ & $<\mathbf{0 . 0 0 1}$ \\
Tumour size & $0.248(0.045-0.431)$ & $\mathbf{0 . 0 1 4}$ \\
CEA & $0.020(-0.185$ to 0.224$)$ & 0.845 \\
CAI9-9 & $-0.038(-0.241$ to 0.168$)$ & 0.708 \\
\hline
\end{tabular}

Abbreviations: $L A T I=$ L-type amino-acid transporter I; CEA = carcinoembryonic antigen; CAI9-9 = carbohydrate antigen 19-9; VEGF= vascular endothelial growth factor; $95 \mathrm{Cl}=95 \%$ confidence interval. Bold values indicate a statistically significant difference.

Table 2 Patient's demographics according to LATI expression

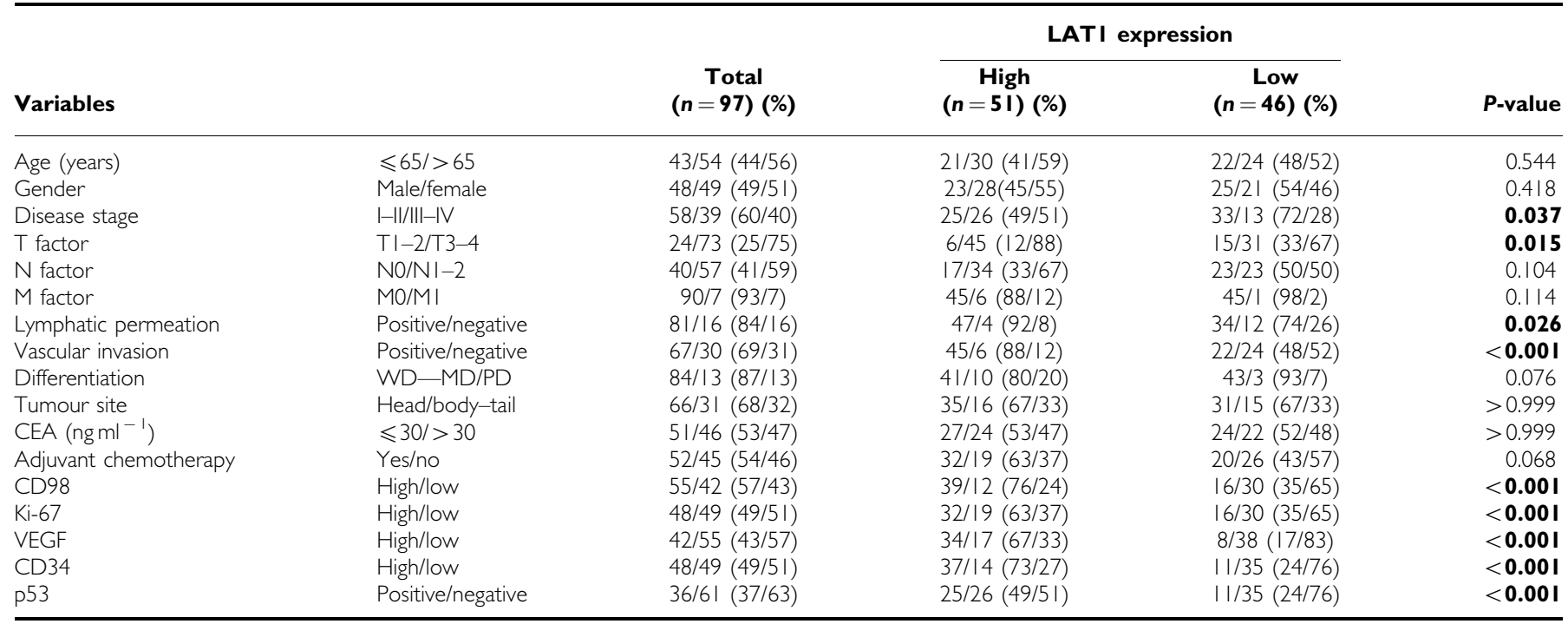

Abbreviations: $L A T I=$-type amino-acid transporter I; $C E A=$ carcinoembryonic antigen; $V E G F=$ vascular endothelial growth factor; $W D=$ well differentiated; $\mathrm{MD}=$ moderately differentiated; $\mathrm{PD}=$ poorly differentiated. Bold values indicate a statistically significant difference. 
Table 4 Univariate and multivariate analysis in progression-free survival

\begin{tabular}{|c|c|c|c|c|c|c|}
\hline Variables & & \multicolumn{2}{|c|}{ Univariate analysis } & \multicolumn{3}{|c|}{ Multivariate analysis } \\
\hline Age (years) & $\leqslant 65 />65$ & $27.9 / 23.4$ & 0.910 & & & \\
\hline Disease stage & $|-||/|||-\mid V$ & $35.3 / 15.4$ & 0.003 & 1.223 & $0.946-1.607$ & 0.122 \\
\hline CEA $\left(n g m l^{-1}\right)$ & $\leqslant 30 />30$ & $29.9 / 20.5$ & 0.121 & & & \\
\hline CAI9-9 $\left(\mathrm{Uml}^{-1}\right)$ & $\leqslant 1321>132$ & $36.3 / 18.1$ & 0.110 & & & \\
\hline Vascular invasion & $\mathrm{P} / \mathrm{N}$ & 15.0/49.6 & $<0.001$ & 1.397 & $0.789-2.386$ & 0.243 \\
\hline Adjuvant chemotherapy & Yes/no & $25.4 / 27.4$ & 0.878 & & & \\
\hline LATI & High/low & $0.0 / 45.3$ & $<0.001$ & 1.226 & $0.873-1.734$ & 0.241 \\
\hline CD98 & High/low & $17.8 / 37 . \mid$ & $0.07 \mid$ & & & \\
\hline $\mathrm{Ki}-67$ & High/low & $0.0 / 41.0$ & $<0.001$ & 1.835 & $0.936-3.792$ & 0.077 \\
\hline VEGF & High/low & $8.5 / 38.2$ & $<0.001$ & 1.132 & $0.842-1.54 \mid$ & 0.415 \\
\hline CD34 & High/low & $17.6 / 34.0$ & 0.582 & & & \\
\hline
\end{tabular}

Abbreviations: $M / F=$ male/female; LATI = L-type amino-acid transporter I; CEA = carcinoembryonic antigen; CAI9-9= carbohydrate antigen I9-9; VEGF=vascular endothelial growth factor, $\mathrm{P} / \mathrm{N}=$ positive/negative; $\mathrm{Cl}=$ confidence interval. Bold values indicate a statistically significant difference.

Table 5 Univariate and multivariate analysis in overall survival

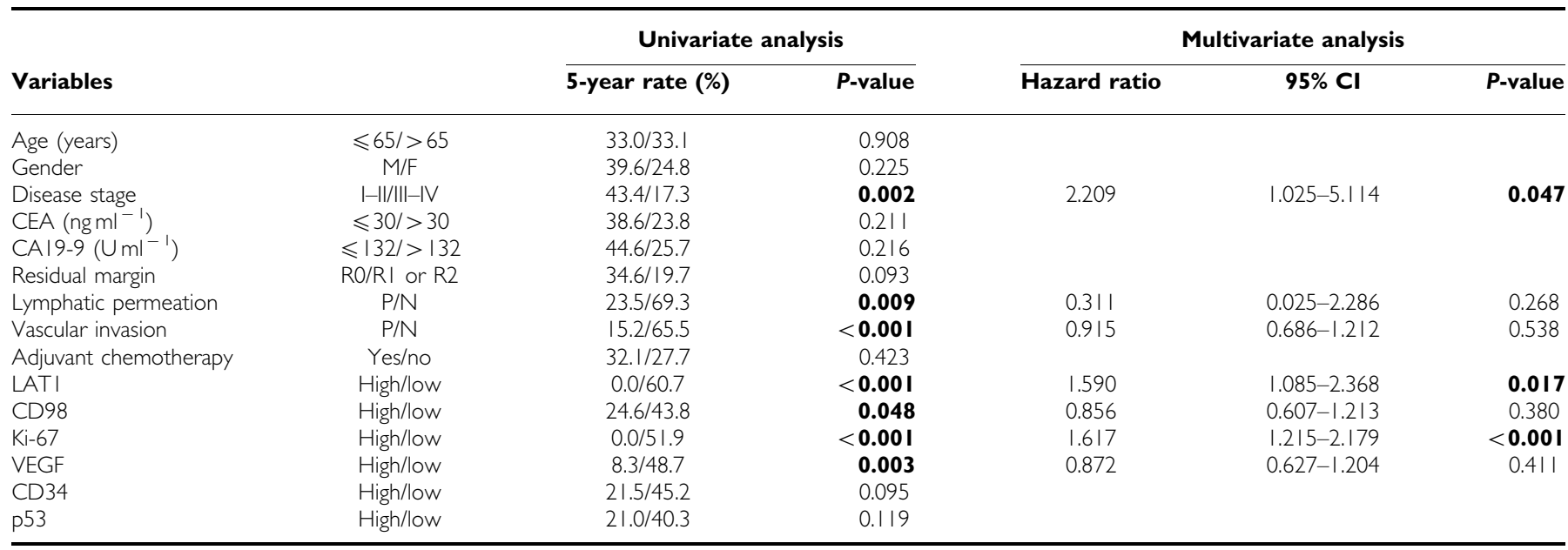

Abbreviations: $\mathrm{M} / \mathrm{F}=$ male/female; LATI = L-type amino-acid transporter I; CEA = carcinoembryonic antigen; CAI9-9=carbohydrate antigen 19-9; VEGF=vascular endothelial growth factor; $\mathrm{P} / \mathrm{N}=$ positive/negative; $\mathrm{Cl}=$ confidence interval. Bold values indicate a statistically significant difference.

predicting poor outcome in patients with surgically resected pancreatic cancer. Moreover, our study showed that LAT1 expression was significantly associated with disease stage, tumour size, tumour proliferation (Ki-67), angiogenesis (VEGF and CD34), the cell cycle regulator p53 and CD98 expression. CD98 has a crucial role in the functional expression of LAT1; therefore, LAT1 directly correlated with the expression of CD98. A recent study has documented that CD98 expression within tumour cells significantly correlates with angiogenesis (VEGF and CD34) and p53 (Kaira et al. 2010b). As LAT1 expression is significantly associated with cell proliferation (Ki-67) in various neoplasms (Kaira et al, 2008; Furuya et al. 2012), we hypothesised that LAT1 expression within pancreatic tumour cells also directly correlated with these molecular markers.

The expression of LAT1 is tumour-specific and has been shown to have essential roles in cell growth and survival. Recent studies have shown that high LAT1 expression is significantly associated with cell proliferation, angiogenesis and poor outcome in patients with various human neoplasms (Kobayashi et al, 2005; Nawashiro et al, 2006; Nakanishi et al, 2007; Kaira et al, 2008; Sakata et al, 2009; Ichinoe et al, 2011). However, little is known regarding the clinical significance of LAT1 expression in various human cancers, such as pancreatic cancer, colorectal cancer, skin tumours (melanoma), sarcoma, head and neck cancer, hepatic cancer and malignant lymphoma. In the present study, LAT1 was highly expressed in pancreatic cancer cells compared with non-malignant pancreatic cells, and the high expression of LAT1 protein within pancreatic cancer cells closely correlated with dismal postoperative survival regardless of adjuvant chemotherapy. One in vitro study has also documented that LAT1 expression in Colo357 cells (human ductal pancreatic AC cells) is higher than that in nonmalignant controls (von Forstner et al, 2011). Interestingly, LAT1 closely correlated with the expression of CD98 in pancreatic malignant and benign cells, but the expression of LAT1 within non-malignant cells did not significantly correlate with angiogenesis, cell proliferation or cell cycle regulation. Our results indicate that CD98 is essential for the functional expression of LAT1 in malignant and non-malignant cells. However, CD98 expression was absent in $6(11.7 \%)$ of 51 patients with high LAT1 expression. Our results suggest that not all patients with pancreatic AC have cooperative expression of LAT1 with CD98, but the reason for the discrepancy in their expression remains unknown. The expression of CD98 has been documented to be elevated in a variety of carcinomas and to have a crucial role in tumour progression 

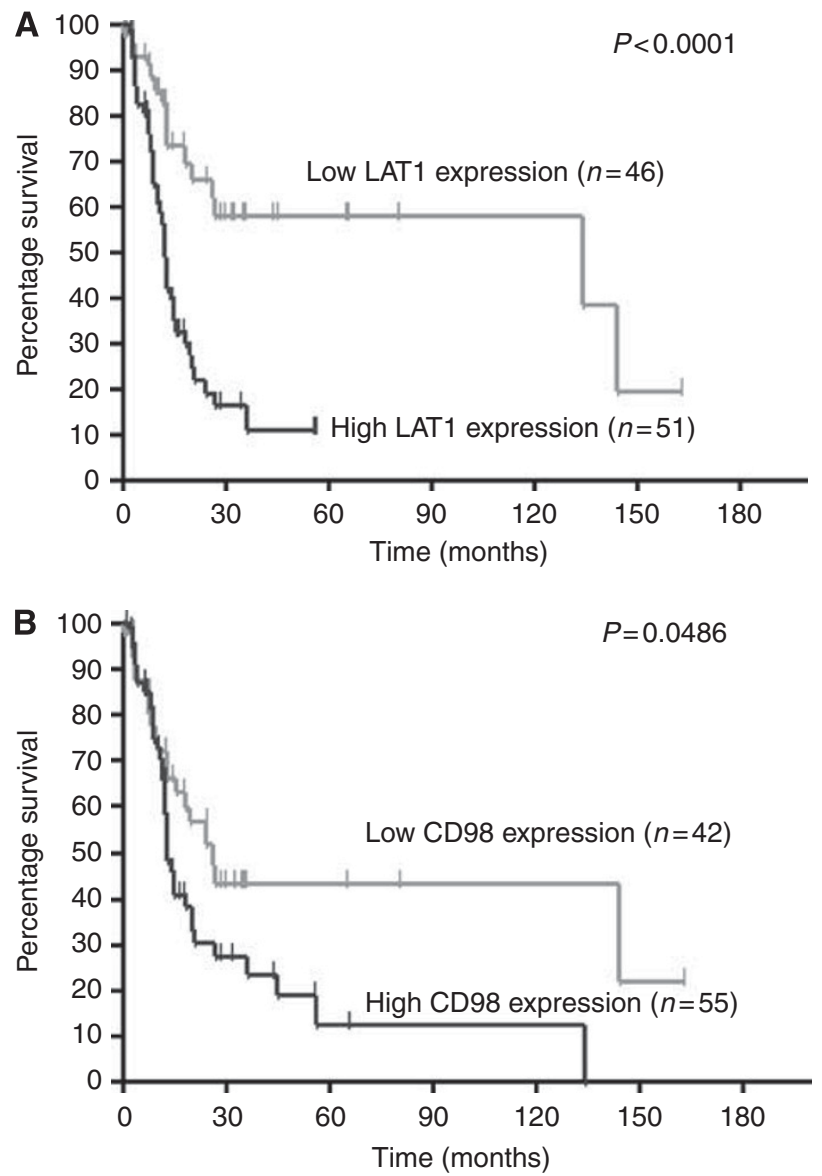

Figure 2 Kaplan-Meier analysis of OS according to LATI (A) and CD98 expression (B). A statistically significant difference in OS was observed between the patients with high LATI and those with low LATI tumour expression $(P<0.00 \mathrm{I})$, and between the patients with high and low CD98 tumour expression $(P=0.0486)$.

and metastasis of human neoplasms; CD98 expression could also represent a significant prognostic factor for predicting poor outcome in lung cancer (Kaira et al, 2009). However, our study did not identify CD98 expression as a significant independent prognostic marker in pancreatic cancer. As CD98 is frequently expressed in benign pancreatic diseases, CD98 may not be a tumour-specific marker in pancreatic tumours.

A recent review has summarised the immunohistochemical biomarkers with prognostic significance in patients with pancreatic cancer and concluded that none of the molecular markers can be recommended for routine clinical use (Ansari et al, 2011). However, several small studies have identified the Ki-67 index as an independent predictor of survival in pancreatic cancer (Linder et al, 1997; Shyr et al, 1999; Yamamoto et al, 2004; Karamitopoulou et al, 2010). Among the prognostic factors Ki-67, p21, p27 and p53, only the Ki-67 index has been described to show

\section{REFERENCES}

Ahrendt SA, Brown HM, Komorowski RA, Zhu YR, Wilson SD, Erickson BA, Erickson BA, Ritch PS, Pitt HA, Demeure MJ (2000) p21 WAF1 expression is associated with improved survival after adjuvant chemoradiation for pancreatic cancer. Surgery 128: 520-530

Ai KX, Lu LY, Huang XY, Chen W, Zhang HZ (2008) Prognostic significance of S100A4 and vascular endothelial growth factor expression in pancreatic cancer. World J Gastroenterol 14: 1931-1935 prognostic significance in 77 patients with pancreatic ductal AC by multivariate analysis (Yamamoto et al, 2004). Moreover, two immunohistochemical studies have demonstrated a significant correlation between p53-positive tumours and poor outcome (Linder et al, 1997; Ahrendt et al, 2000), 3 of 18 studies have identified VEGF as an independent prognostic marker (Ikeda $e t a l$, 1999; Sun et al, 2007; Ai et al, 2008), and only CD34 has been described to be associated with outcome by multivariate analysis in two studies (Ikeda et al, 1999; Fujioka et al, 2001). However, other studies have found no relationship between these molecular markers and survival in pancreatic cancer (Ansari et al, 2011). Therefore, whether the presence of these molecular markers has any prognostic implications remains unclear. The results of our study identified the Ki-67 index as an independent prognostic factor for predicting poor outcome. The level of cell proliferation may have a significant prognostic implication in tumour cells with high LAT1 expression. Although a recent retrospective study has demonstrated that patients with adjuvant therapy have more adverse prognostic factors than those without adjuvant therapy (Corsini and Miller, 2008), LAT1 and the Ki-67 index were associated with prognostic significance regardless of adjuvant therapy. However, the prognostic role of Ki-67 varies between different studies. The expression profile of Ki-67 differs between the studies, and the methods used in the studies also vary. Therefore, optimisation and standardisation of these molecular techniques is necessary. Further study is warranted to confirm our results using a large number of patients with pancreatic cancer.

The limitations of this study must be addressed. Our study analysed the outcome of subgroups based on high and low LAT1 expression. As this study analysed the survival of small groups, the subgroup analysis may bias our results. Further study is warranted to analyse a large number of patients with pancreatic cancer and to investigate the optimal cutoff points for the expression level of LAT1.

In conclusion, high expression of LAT1 (measured by immunostaining) can serve as an independent prognostic marker to predict poor outcome after surgical resection and may be an important clinical marker of therapy for pancreatic AC. Inhibition of LAT1 function may arrest tumour growth, and LAT1 represents an attractive target for adjuvant therapy in the future.

\section{ACKNOWLEDGEMENTS}

This work was supported in part by Grant 23591750 (KK) from the Ministry of Education, Culture, Sports, Science and Technology, Japan, and the National Hospital Organisation Policy Based Medical Services. We thank Ms Masako Saito for technical assistance with the immunohistochemical analysis.

\section{Conflict of interest}

The authors declare no conflict of interest.

Supplementary Information accompanies the paper on British Journal of Cancer website (http://www.nature.com/bjc)
Ansari D, Rosendahl A, Elebro J, Andersson R (2011) Systematic review of immunohistochemical biomarkers to identify prognostic subgroups of patients with pancreatic cancer. Br J Surg 98: 1041-1055

Cameron JL, Riall TS, Coleman J, Belcher KA (2006) One thousand consecutive pancreaticoduodenectomies. Ann Surg 244: 10-15

Corsini MM, Miller RC, Haddock MG, Donohue JH, Farnel MB, Nagorney DM, Jatoi A, McWilliams RR, Kim GP, Bhatia S, Iott MJ, 
Gunderson LL (2008) Adjuvant radiotherapy and chemotherapy for pancreatic carcinoma: the Mayo Clinic Experience (1975-2005). J Clin Oncol 26: 3511-3516

Christensen HN (1990) Role of amino acid transport and countertransport in nutrition and metabolism. Physiol Rev 70: 43-77

Delcore R, Rodriguez FJ, Forster J, Hermreck AS, Thomas JH (1996) Significance of lymph node metastases in patients with pancreatic cancer undergoing curative resection. Am J Surg 172: 463-468

Fuchs BC, Bode BP (2006) Amino acid transporters ASCT2 and LAT1 in cancer: partners in crime? Semin Cancer Biol 15: 254-266

Fujioka S, Yoshida K, Yanagisawa S, Kawakami M, Aoki T, Yamazaki Y (2001) Angiogenesis in pancreatic carcinomas: thymidine phosphorylase expression in stromal cells and intratumoral microvessel density as independent predictors of overall and relapse-free survival. Cancer 92: 1788-1797

Furuya M, Horiguchi J, Nakajima H, Kanai Y, Oyama T (2012) Correlation of L-type amino acid transporter 1 and CD98 expression with triple negative breast cancer prognosis. Cancer Sci 103: 382-389

Geer RJ, Brennan MF (1993) Prognostic indicators for survival after resection of pancreatic adenocarcinoma. Am J Surg 165: 68-72

Hellan M, Sun CL, Artinyan A, Mojica-Manosa P, Bhatia S, Ellenhorn JD, Kim J (2008) The impact of lymph node number on survival in patients with lymph node-negative pancreatic cancer. Pancreas 37: 19-24

Ichinoe M, Mikami T, Yoshida T, Igawa I, Tsuruta T, Nakada N, Anzai N, Suzuki Y, Endou H, Okayasu I (2011) High expression of L-type aminoacid transporter 1 (LAT1) in gastric carcinomas: comparison with non-cancerous lesions. Pathol Int 61: 281-289

Ikeda N, Adachi M, Taki T, Huang C, Hashida H, Takabayashi A, Sho M, Nakajima Y, Kanehiro H, Hisanaga M, Nakano H, Miyake M (1999) Prognostic significance of angiogenesis in human pancreatic cancer. Br J Cancer 79: 1553-1563

Jemal A, Siegel R, Ward E, Hao Y, Xu J, Murray T, Thun MJ (2008) Cancer statistics, 2008. CA Cancer J Clin 58: 71

Kaira K, Oriuchi N, Imai H, Shimizu K, Yanagitani N, Sunaga N, Hisada T, Tanaka S, Ishizuka T, Kanai Y, Endou H, Nakajima T, Mori M (2008) Prognostic significance of L-type amino acid transporter 1 expression in resectable stage I-III nonsmall cell lung cancer. Br J Cancer 98: 742-748

Kaira K, Oriuchi N, Imai H, Shimizu K, Yanagitani N, Sunaga N, Hisada T, Kawashima O, Kamide Y, Ishizuka T, Kanai Y, Nakajima T, Mori M (2009) CD98 expression is associated with poor prognosis in resected non-small-cell lung cancer with lymph node metastases. Ann Surg Oncol 16: 3473-3481

Kaira K, Endo M, Abe M, Nakagawa K, Ohde Y, Okumura T, Takahashi T, Murakami H, Tsuya A, Nakamura Y, Naito T, Hayashi I, Serizawa M, Koh Y, Hanaoka H, Tominaga H, Oriuchi N, Kondo H, Nakajima T, Yamamoto N (2010a) Biologic correlation of $2-\left[{ }^{18} \mathrm{~F}\right]-$ fluoro-2-deoxy-Dglucose uptake on positron emission tomography in thymic epithelial tumors. J Clin Oncol 28: 3746-3753

Kaira K, Takahashi T, Abe M, Akamatsu H, Nakagawa K, Ohde Y, Okumura T, Murakami H, Tsuya A, Nakamura Y, Naito T, Kondo H, Nakajima T, Endo M, Yamamoto N (2010b) CD98 expression is associated with the grade of malignancy in thymic epithelial tumors. Oncol Rep 24: 861-867

Kaira K, Oriuchi N, Takahashi T, Nakagawa K, Ohde Y, Okumura T, Murakami H, Shukuya T, Kenmotsu H, Naito T, Kanai Y, Endo M, Kondo H, Nakajima T, Yamamoto N (2011a) LAT1 expression is closely associated with hypoxic markers and mTOR in resected non-small cell lung cancer. Am J Transl Res 3: 468-478

Kaira K, Oriuchi N, Takahashi T, Nakagawa K, Ohde Y, Okumura T, Murakami H, Shukuya T, Kenmotsu H, Naito T, Kanai Y, Endo M,
Kondo H, Nakajima T, Yamamoto N (2011b) L-type amino acid transporter 1 (LAT1) expression in malignant pleural mesothelioma. Anticancer Res 31: 4075-4082

Kanai Y, Segawa H, Miyamoto K, Uchino H, Takeda E, Endou H (1998) Expression cloning and characterization of a transporter for large neutral amino acids activated by the heavy chain of 4F2 antigen (CD98). J Biol Chem 273: 23629-23632

Karamitopoulou E, Zlobec I, Tornillo L, Carafa V, Schaffner T, Brunner T, Borner M, Diamantis I, Zimmermann A, Terracciano L (2010) Differential cell cycle and proliferation marker expression in ductal pancreatic adenocarcinoma and pancreatic intraepithelial neoplasia (Pan IN). Pathology 42: 229-234

Kobayashi H, Ishii Y, Takayama T (2005) Expression of L-type amino acid transporter 1 (LAT1) in esophageal carcinoma. J Surg Oncol 90: 233-238

Linder S, Parrado C, Falkmer UG, Blasjo M, Sundelin P, von Rosen A (1997) Prognostic significance of Ki-67 antigen and p53 protein expression in pancreatic duct carcinoma: a study of the monoclonal antibodies MIB-1 and DO-7 in formalin-fixed paraffin-embedded tumour material. Br J Cancer 76: 54-59

McGivan JD, Pastor-Anglada M (1994) Regulatory and molecular aspects of mammalian amino acid transport. Biochem J 299: 321-334

Nakanishi K, Ogata S, Matsuo H, Kanai Y, Endou H, Hiroi S, Tominaga S, Aida S, Kasamatsu H, Kawai T (2007) Expression of LAT1 predicts risk of progression of transitional cell carcinoma of the upper urinary tract. Virchows Arch 451: 681-690

Nawashiro H, Otani N, Shinomiya N, Fukui S, Ooigawa H, Shima K, Matsuo H, Kanai Y, Endou H (2006) L-type amino acid transporter 1 as a potential molecular target in human astrocytic tumors. Int J Cancer 119: 484-492

Sakata T, Ferdous G, Tsuruta T, Satoh T, Baba S, Muto T, Ueno A, Kanai Y, Endou H, Okayasu I (2009) L-type amino acid transporter 1 as a novel biomarker for high-grade malignancy in prostate cancer. Pathol Int 59: 7-18

Shyr YM, Su CH, Li AF, Wu CW, Lui WY (1999) The role of MIB-1 index in the prognosis of resectable pancreatic head cancer. Hepatogastroenterology 46: 2968-2973

Sobin LH, Gospodarowicz MK, Ch Wittekind (2009) (eds) International Union Against Cancer (UICC) TNM Classification of Malignant Tumors. 7th edn. Wiley-Blackwell: Oxford, UK

Sun HC, Qiu ZJ, Liu J, Sun J, Jiang T, Huang KJ, Yao M, Huang C (2007) Expression of hypoxia-inducible factor-1 alpha and associated proteins in pancreatic ductal adenocarcinoma and their impact on prognosis. Int J Oncol 30: 1359-1367

von Forstner C, Zuhayra M, Ammerpohl O, Zhao Y, Tiwari S, Jansen O, Kalthoff H, Henze E, Egberts JH (2011) Expression of L amino acid transporter system 1 and analysis of iodine-123-methyltyrosine tumor uptake in a pancreatic xenotransplantation model using fused highresolution-micro-SPECT-MRI. Hepatobiliary Pancreat Dis Int 10: 30-37

Yamamoto S, Tomita Y, Hoshida Y, Morooka T, Nagano H, Dono K, Umeshita K, Sakon M, Ishikawa O, Ohigashi H, Nakamori S, Monden M, Aozasa K (2004) Prognostic significance of activated Akt expression in pancreatic ductal adenocarcinoma. Clin Cancer Res 10: 2846-2850

Yanagida O, Kanai Y, Chairoungdua A, Kim DK, Segawa H, Nii T, Cha SH, Matsuo H, Fukushima J, Fukasawa Y, Tani Y, Taketani Y, Uchino H, Kim JY, Inatomi J, Okayasu I, Miyamoto K, Takeda E, Goya T, Endou H (2001) Human L-type amino acid transporter 1 (LAT 1): characterization of function and expression in tumor cell lines. Biochim Biophys Acta 1514: $291-302$

This work is published under the standard license to publish agreement. After 12 months the work will become freely available and the license terms will switch to a Creative Commons Attribution-NonCommercial-Share Alike 3.0 Unported License. 